\title{
Wotistische Überraschung versteckt sich über die Jahrtausendwende
}

Barbera, Manuel. 2013 (1994). A short etymological glossary of the Votic language. Città di Castello (PG): I libri di Emil. LXIX + 426 S. Barbera, Manuel. 2012 (1995). Introduzione storico-descrittiva alla lingua vota (fonologia e morfologia). Città di Castello (PG): I libri di Emil. 448 S.

Bücher mehr als ein Vierteljahrhundert nach der Veröffentlichung $\mathrm{zu}$ rezensieren ist ungewöhnlich. Die Verzögerung aber beweist nur, wie lange es diesem merkwürdigen Zwillingspaar gelungen ist, sich vor der uralistischen Forschergemeinschaft zu verstecken. Manuel Barbera, heute Professor der Didaktik der Gegenwartssprachen an der Universität Turin, hat viel über mehrere Sprachfamilien publiziert; auf dem Gebiet der Uralistik s. z.B. Barbera (1993a, 1993b, 1999).

Beide hier rezensierten Werke behandeln das Westwotische. Ursprünglich beabsichtigte der Autor mit seinem etymologischen Wörterbuch zu promovieren, aber dies war dem sprachwissenschaftlichen Institut der Universität Pavia nicht recht, so dass Barbera noch die Introduzione storico-descrittiva verfasste.

Die Periode der Veröffentlichung zeigt sich auf lustige Weise im Layout der Werke. Die Schriften sind offenbar für den Druck mit einem Tintenstrahldrucker ausgewählt, und die meisten Diakritika hat der Autor rechts von seinen Buchstaben platzieren müssen. Auch beim Druck sind Missgeschicke passiert. Beim etymologischen Wörterbuch fehlt bedauerlicherweise Seite 107, an deren Stelle Seite 120 ein zweites Mal gedruckt wurde.

\section{Die historisch-deskriptive Grammatik}

Das Werk ist nicht ganz, was man dem Namen nach davon erwarten könnte, sondern vielmehr eine vergleichende Grammatik der ostseefinnischen Sprachen aus der Perspektive des Wotischen. Dies ist selbstverständlich eine willkommene Abwechslung zum traditionellen Fennozentrismus der Disziplin. Die historische Dimension steht stellenweise stärker, stellenweise weniger im Vordergrund, vor 
allem aber bei der Darstellung der Kasusflexion, die alle finnisch-permischen Sprachen umfasst und innerhalb des Buches gewissermaßen eine separate Studie bildet.

Wertvoll ist die Behandlung der westwotischen Dialekte S. 60-71, für die Barbera aus Aristes (1968) Grammatik alle für einzelne Dörfer erwähnten lautlichen, morphologischen und lexikalischen Züge gesammelt und diese zu Verzeichnissen über Dialektzüge der jeweiligen Dörfer arrangiert hat. Diese Verzeichnisse wären ein guter Ausgangspunkt auch für eine umfangreichere Darstellung der Dialekte des Westwotischen.

Angebracht ist die indoeuropäistische Kritik an Postis Theorie zum Ursprung des Stufenwechsels sowie die Erklärung des „suffixalen Stufenwechsels" als ein vom Stufenwechsel separates Phänomen S. 146. Eine gute Innovation ist auch die Reihenfolge der Behandlung der Themen, so werden zum Beispiel bei der Darstellung des Lautsystems die neuen, „marginalen“ Konsonanten nicht erst zum Schluss erwähnt, sondern gleich am Anfang behandelt.

Das Werk hätte einer Vorkontrolle durch einen erfahrenen Uralisten bedurft, denn die Menge der Fehler wirkt sich störend aus. Die meisten davon sind klein, aber einige verursachen doch Probleme im Hinblick auf das Gesamtbild. S. 91, 94 postuliert der Verfasser ohne Begründung für das Urfinnische ein $\ddot{o}$ in nichtersten Silben, das im Wotischen geschwunden sei. Eine fehlerhafte urfi. Rekonstruktion ${ }^{\star}$ onne-ton (statt -ťton) S. 145 führt zu einer falschen Schlussfolgerung bei der Bewertung der Entwicklung des Konsonantismus nach unbetonter Silbe.

Ein erheblicher Mangel auf Seiten der Lautlehre ist die minimale Behandlung der Diphthonge S. 9798. Diphthonge in nicht-ersten Silben erwähnt der Verfasser überhaupt nicht. Im Bereich der Formenlehre bleibt die Rekonstruktion der wotischen Terminativendung urfi. ${ }^{*} n$-sak S. 167 ohne Erklärung.

Der Autor ist gelegentlich etwas $\mathrm{zu}$ eifrig mit seiner Zeit gegangen und hat ohne Kritik Hypothesen übernommen, die zum Zeitpunkt seiner Arbeit frisch waren, wie etwa die Verwandtschaft des Jukagirischen mit den uralischen Sprachen, die vierte Längenstufe estnischer Silben und den teilweise ururalischen Ursprung der im Ostseefinnischen anzutreffenden Vergangenheitsmarkers -si- (S. 306).

Den Dialekt von Kukkuzi und das Krewinische fasst Barbera z.B. S. 51, 120-22 als Südwestwotisch zusammen, was offenbar ausschließlich auf Postis (1934) Auffassung zurückgeht, dass beide hinsichtlich der Entwicklung ${ }^{\star} k>{ }^{*} c$ archaisch seien. Wenn wir jedoch dem S. 37 von Viitso übernommenen Schema 
(vgl. Viitso 2008: 64) folgen, stammt der Dialekt von Kukkuzi aus demselben Zweig wie das Ingrische, was den Erhalt von urfi. ${ }^{*} k$ dort besser erklärt (so auch Winkler 1997: 426).

\section{Das etymologische Wörterbuch}

Dieses besondere Werk hat die bisherige komplette Nichtbeachtung nicht verdient. Es ist besser gelungen als die Grammatik. Eines seiner erklärten Hauptziele, die Darstellung des gesamten in gedruckten Quellen bekannten Wortschatzes des Westwotischen, hat zwar nach dem Erscheinen von VKS seine Bedeutung verloren, was auch der $\mathrm{Au}$ tor selber in seinem Nachwort eingesteht. Seine Ziele und Praktiken auf dem Gebiet der wotischen Lexikographie hat Barbera auch in zwei Artikeln (2001, 2002) dargestellt.

Auch dass die Arbeit am Werk mitten in das zweite goldene Zeitalter der uralistischen Lehnwortforschung fiel, hat das Wörterbuch schnell veralten lassen, wenngleich es gerade im Bereich der Entlehnungen am stärksten ist. Das bedeutendste Verdienst des Buches ist die gründliche Besprechung der mutmaßlichen indogermanischen Ausgangsformen angenommener Lehnwörter in den Lemmata.

Einem Uralisten können die Wortartikel übertrieben lang wirken, insbesondere im Fall junger
Entlehnungen aus dem Russischen, aber hierzu trägt die Tradition seiner eigenen etymologischen Wörterbücher bei, deren Hauptaugenmerk auf den alten Erbwörtern und deren Entsprechungen liegt. Barbera stellt diese Konstellation auf den Kopf. Sein logischer Ausgangspunkt ist die gleichmäßig exakte Beschreibung aller hinreichend sicheren Informationen über die Hintergründe eines Wortes, unabhängig davon, in welcher Sprachfamilie es auftritt.

Dank seiner Vertrautheit mit der indogermanistischen Forschungsliteratur gelingt die Analyse mutmaßlicher Entlehnungen - beispielsweise kańevo, Narva, têrva (fi. terva) und terve - Barbera besser als jedem etymologischen Wörterbuch der ostseefinnischen Sprachen, abgesehen von LägLoS. Für das Wort irsi (fi. hirsi) legt Barbera eine ganz neue, interessante baltische Erklärung vor. Mehrere Wörter unterschiedlichen Alters, die aus derselben indogermanischen Wortsippe, aber aus verschiedenen Quellen entlehnt wurden, verbindet der Autor vorbildlich mit Querverweisen untereinander.

Dementsprechend scheitert das Werk am deutlichsten an der Erklärung solcher Wörter, bei der eine Hintergrunduntersuchung der als Ausgangsformen einer Entlehnung angesetzten Wörter nicht stattfindet. Beispiele hierfür sind mettsä (fi. metsä), ümpäri (fi. ympäri) und 
varsi. Bei der Bewertung vorgeschlagener Entsprechungen aus entfernt verwandten Sprachen hat sich der Autor als Nicht-Uralist vor allem auf UEW und Collinder gestützt und bietet daher kaum Neues.

Insgesamt hätte Barbera sich mit seiner Sachkenntnis seinerzeit hervorragend geeignet, die SSA-Redaktion zu vervollständigen, deren Stärken auf dem Gebiet des uralischen Lexikons lagen. Ohne Verbindungen zur uralistischen etymologischen Gemeinschaft wurde das wotische etymologische Wörterbuch jedoch zu einem Nachschlagewerk anderer Art, das auf seine eigene, interessante Weise die Standardquellen der ostseefinnischen Etymologie ergänzt und ist mit seinen umfangreichen indoeuropäistischen Literaturangaben vor allem für die Lehnwortforschung hilfreich.

\section{Santeri Junttila}

\section{Literatur}

Ariste, Paul. 1968. A grammar of the Votic language. Bloomington \& The Hague: Indiana University \& Mouton.

BArberA, MANuel. 1993a. La gradazione baltofinnica. Londra: Lothian Foundation Press.

Barbera, Manuel. 1993b. Problemi di ricostruzione nel consonantismo uralico. Ponto-Baltica V. 7-6o.

Barbera, Manuel. 1999. Appunti su definitezza e partitivo nelle lingue baltofinniche. Bollettino dell'Atlante linguistico italiano $3 \mathrm{a}$ serie XXIII. 119-188.
Barbera, Manuel. 2001. Providing a future for a disappearing language: Some notes on Votic lexicography. Linguistica Lettica VII. 180-213.

BARbera, MANuel. 2002. Formats of etymology: the case of 'beer' and 'juniper' in Votic. In Proceedings of the Tenth EURALEX International Congress, Copenhagen, Denmark, August 13-17 2002. Vol. II. 563-568. Copenhagen: Center for Sprogteknologi.

Collinder, BJörn. 1955. Fenno-Ugric vocabulary: An etymological dictionary of the Uralic languages. Stockholm: Almqvist \& Wiksell.

LägLoS I-III = KYlstra, A. D. \& HAHmo, SirkKA-LiIsA \& HofSTRA, Tette \& Nikкilä, Osmo. 19912012. Lexikon der älteren germanischen Lehnwörter in den ostseefinnischen Sprachen 1-3. Amsterdam \& Atlanta: Rodopi.

Posti, LAURI. 1934. Vatjan kielen $k>t \check{s}$ äänteenmuutoksen iästä. Kalevalaseuran vuosikirja 14. 139-148.

SSA = ITKONEN, ERKKI \& KULONEN, Ulla-Maija (Hrsg.). 1992-2000. Suomen sanojen alkuperä. Etymologinen sanakirja 1-3. Helsinki: Kotimaisten kielten tutkimuskeskus \& Suomalaisen Kirjallisuuden Seura.

UEW = RÉDEI, KÁROLY (Hrsg.). 1988. Uralisches etymologisches Wörterbuch I-II. Wiesbaden: Harrassowitz. Virtso, Tirt-Rein. 2008. Liivi keel ja läänemeresoome keelemaastikud. Tartu \& Tallinn: Eesti keele sihtasutus.

VKS = AdLER, ElNA \& GRÜNBERG, SILJA (Hrsg.). 1990-2011. Vadja keele sõnaraаmat. Словарь водского языка 1-7. Tallinn: Eesti Teaduste Akadeemia Keele ja Kirjanduse Instituut.

WINKLER, EBERHARD. 1997. Krewinisch: Zur Erforschung einer ausgestorbenen ostseefinnischen Sprachform. Wiesbaden: Harrassowitz. 